\title{
Finite Element Model Modification of Arch Bridge Based on Radial Basis Function Neural Network
}

\author{
Tongqing Chen ${ }^{1}$, Lei Wang ${ }^{1 *}$, Xijuan Jiang ${ }^{1}$, Yubin Wang ${ }^{1}$ and Kai Yan $^{2}$ \\ ${ }^{1}$ School of Civil Engineering and Architecture, University of Jinan, Jinan 250022, China. \\ ${ }^{2}$ School of Civil Engineering, Shandong Jianzhu University, Jinan 250101, China.
}

\begin{abstract}
Compared with other neural networks, Radial Basis Function (RBF) neural network has the advantages of simple structure and fast convergence. As long as there are enough hidden layer nodes in the hidden layer, it can approximate any non-linear function. In this paper, the finite element model of a through tied arch bridge is modified based on Neural Network. The approximation function of RBF neural network is utilized to fit the implicit function relationship between the response of the bridge and its design parameters. Then the finite element model of the bridge structure is modified. The results show that RBF neural network is efficient to modify the model of a through tied arch bridge.
\end{abstract}

\section{Bridge Overview}

The longitudinal stiffening girder of bridge main span is a single box and five chambers bidirectional prestressed concrete box structure, solid section beam at end. The arch rib is a steel box structure, the transition between arch rib and main girder joints with steel-concrete joints, which is $6.32 \mathrm{~m}$ long. The main span is 247.0807 meters. The vector height is 55.5 meters, and the rise-span ratio is $1 / 4.5$. There are 33 pairs of suspenders on the whole bridge. The distance between the bridge suspender is 7 meters, and the transverse spacing of the bridge is 3.8 meters.

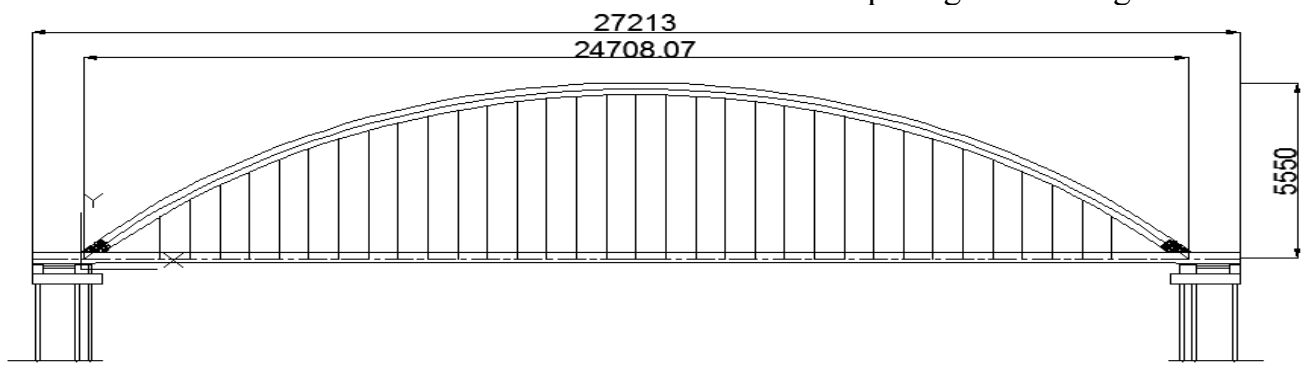

Figure 1. Arch Bridge Overall Layout (Unit: cm)

\section{Mapping mechanism of Radial Basis Function Neural Network}

By calculating the generalization of the distance function between the input and the center of the hidden layer, it maps to the high-dimensional space. This step is accomplished through the radial basis function [1,2]. Gauss function is often used in radial basis function, and its representation is

$$
\varphi=\mathrm{e}^{-\mathrm{d}^{\wedge} 2 /\left(2 \sigma^{\wedge} 2\right)}
$$

Distance $=\left\|x-c_{j}\right\|$. It's the distance between the vector and the center of each hidden layer. Distance means that the closer to the node, the greater the impact of the output of the node. $\sigma$ is a smoothing factor. When $\sigma$ is relatively small. Gauss function will be sharp. The input is mapped to high-dimensional space by Gauss function. The mapping relation for this step is as follows:

$$
h_{j}(x)=\Phi\left(\left\|x-c_{j}\right\|, \sigma_{j}\right)=\exp \left[-\frac{\left\|x-c_{j}\right\|^{2}}{2 \sigma_{j}^{2}}\right]
$$

The basis function is then weighted and output linearly at the output layer, and the mapping relationship is as follows:

$$
f(x)=\sum_{j=1}^{n} h_{j}(x) w_{j}
$$

Among them, $h_{j}(x)$ is the output of the $j$ hidden unit. Vector $x$ is the signal acting on the input layer. $c_{j}$ is the center of the radial basis function of the $j$ hidden element. $\sigma_{j}$ is the base function width. $w_{j}$ is the weight from hidden layer to output layer. Therefore, using radial basis function (RBF) neural network for fitting is actually 
a linear interpolation of weight $w_{j}$ in the range of the center $c_{j}$ and radius width $\sigma_{j}$ of the hidden layer.

\section{Realization of Radial Basis Function Neural Network}

From the above, a RBF neural network is determined by determining the center $c_{j}$ and width $\sigma_{j}$ of the neuron basis function in the hidden layer and the weight $w_{j}$ from the hidden layer to the output layer [3]. The self-organizing center selection method includes the first stage for estimating the hidden layer center and standard deviation and the second stage for learning the weights.

\subsection{Determining Cluster Centers}

Firstly, according to the empirical assumption, there are $I$ clustering centers. Cluster Centers with Multiple Iterations $t_{i}(n), i=1,2, \ldots I$

$t_{i}(n+1)=\left\{\begin{array}{c}t_{i}(n)+\eta\left[X_{K}(n)-t_{i}(n)\right], i=i\left(X_{K}\right) \\ t_{i}(n), \text { Other }\end{array}\right.$

$\eta$ is the learning step, and the scope is as follows: $0<$ $\eta<1$;

\subsection{Determining standard deviation}

If the activation function of hidden layer adopts Gauss radial basis function, the standard deviation can be obtained from the following formula:

$$
\sigma=\frac{d_{\max }}{\sqrt{2 n}}
$$

In the formula, $d_{\max }$ is the maximum distance among the final clustering centers, and $n$ is the number of hidden nodes.

\subsection{Determine the weights between the hidden layer and the output layer}

The calculating formulas of the least square method are as follows:

$$
\omega=\exp \left(\frac{n}{d_{\max }^{2}}\left\|X_{k}-X_{i}\right\|^{2}\right)
$$

Among them, $k=1,2, \cdots, K ; i=1,2, \cdots, I$

\section{Finite Element Model Updating of Arch Bridge}

\subsection{Determination of parameters}

The eighth-order frequencies measured by the bridge are taken as state parameters. That is, the input of the neural network [4]. The elastic modulus and material mass density of bridge structure are selected as design parameters. This article, the elastic modulus and material mass density of the structure are selected as the parameters to be modified. Including elastic modulus $E_{l}$ and density $D_{1}$ of the main beam. Elastic modulus $E_{2}$ and density $D_{2}$ of steel arch ribs and suspenders. Elastic modulus $E_{3}$ and density $D_{3}$ of beam-arch joint. And the elastic modulus $E_{4}$ and density $\mathrm{D}_{4}$ of abutment. Perturbation of repair parameters and frequency-based sensitivity analysis [5]. Table 1 lists the preliminary screened parameters, their original values and perturbation values. Each of the eight parameters to be corrected in the preliminary screening was increased by $10 \%$. The sensitivity of the first, second, third and fifth order vertical frequencies and the first and fourth order torsional frequencies are analysed. The results are shown in Figure 2. The eighth order frequencies are numbered as follows: $f_{1}, f_{2}, f_{3}, \cdots, f_{8}$ 。

Table 1. Initial value and perturbation value of bridge design parameters

\begin{tabular}{ccccccccc}
\hline parameter & $D_{1}$ & $E_{1}$ & $D_{2}$ & $E_{2}$ & $D_{3}$ & $E_{3}$ & $D_{4}$ & $E_{4}$ \\
\hline Original value & 2937 & $3.45 \times 10^{10}$ & 7850 & $2.05 \times 10^{11}$ & 2985 & $3.45 \times 10^{10}$ & 2600 & $3.25 \times 10^{10}$ \\
increases by $10 \%$ & 3230.7 & $3.8 \times 10^{10}$ & 8635 & $2.26 \times 10^{11}$ & 3283.5 & $3.8 \times 10^{10}$ & 2860 & $3.575 \times 10^{10}$ \\
\hline
\end{tabular}

Note: The unit of modulus of elasticity is: $\mathrm{Pa}$, and the unit of density is: $\mathrm{kg} / \mathrm{m} 3$. 


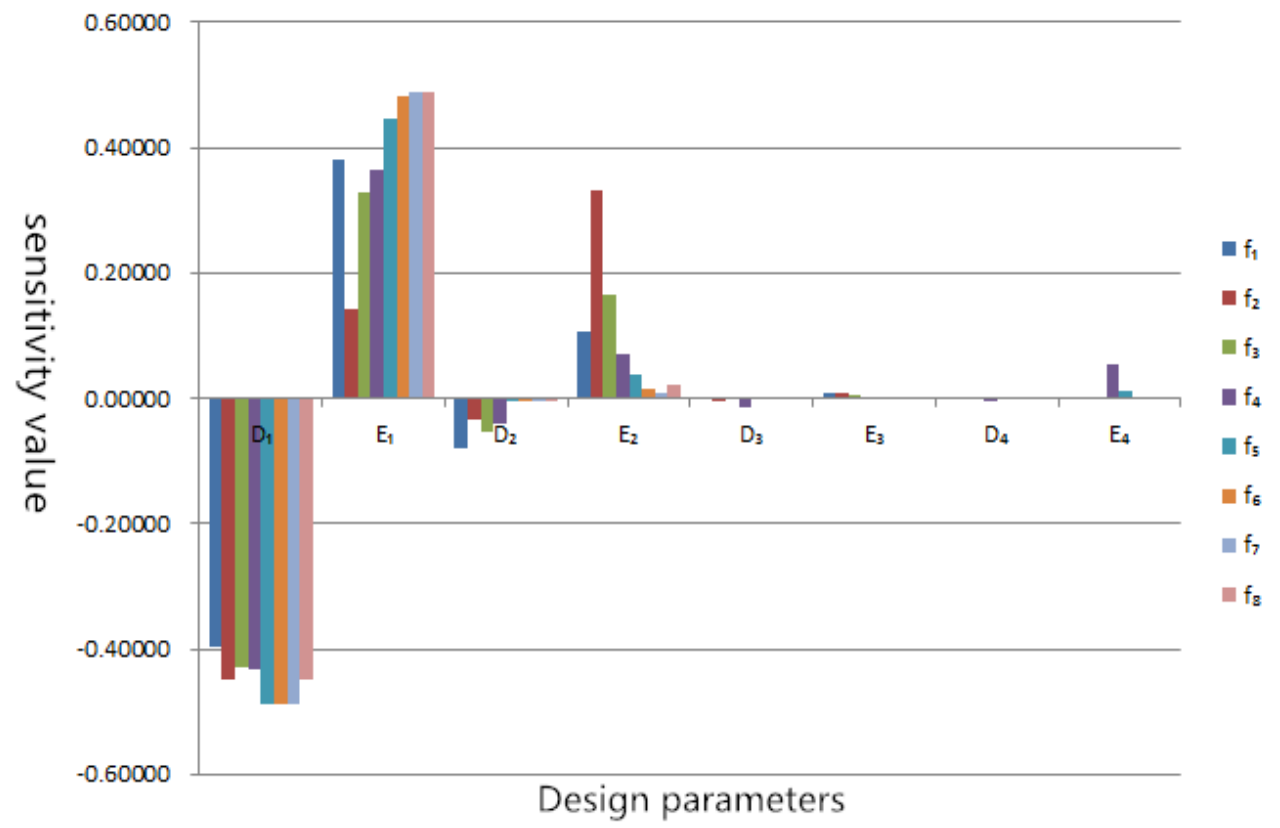

Figure 2. Frequency sensitivity analysis of each parameter

\subsection{Determination of training samples}

In this paper, the density and elastic modulus of steelconcrete composite section at arch foot, concrete and steel bar at main girder and pier abutment are considered as a whole. The design parameters of concrete structural materials are $85 \% \sim 115 \%$ of their initial values. The gradient of the selected design parameters is $1 \%$ in its range of variation, and each parameter has 31 levels. Sample points are determined by uniform design method $[6,7]$. A total of 31 trials are required. There are 31 sets of sample data. The output sample of the neural network is brought into the finite element model to calculate the eighth-order frequency response.

Among them, $f_{1} \sim f_{3}$ is the first third-order vertical bending frequency, $f_{4}$ is the fifth-order vertical bending frequency, and $f_{5} \sim f_{8}$ is the first fourth-order torsional frequency.

\subsection{Building RBF Neural Network}

In this paper, a radial basis function neural network is designed by using the neural network toolbox in
MATLAB. Its function format is as follows: net=newrb (p, t, goal, spread). $P$ and $T$ are input and output samples respectively, and goal is the mean square error of neural network.

spread is the distribution of radial basis function. The input sample is the eighth order frequency obtained by uniform design, so the dimension of the input vector of the neural network is 8 . The output samples include $D_{1}, E_{1}$, $D_{2}, E_{2}, E_{3}$ and $E_{4}$, so they are 6-dimensional vectors. RBF neural network is constructed with 31 sets of sample data obtained by uniform design method. Mean square error of neural network: $1.0 \times 10^{-3}$.Radial basis function (RBF) is selected as the activation function of RBF neural network. The error of the neural network can be minimized by selecting the value of spread in turn. When spread is 0.88 , the network error is the smallest. The error descent curve is shown in Figure 3. At this time, the trained neural network is obtained, as shown in Figure 4. It can be seen from the figure that the training of neural network based on MATLAB. When the center of the hidden layer reaches 50 , the accuracy requirement of the mean square error setting has been achieved. 


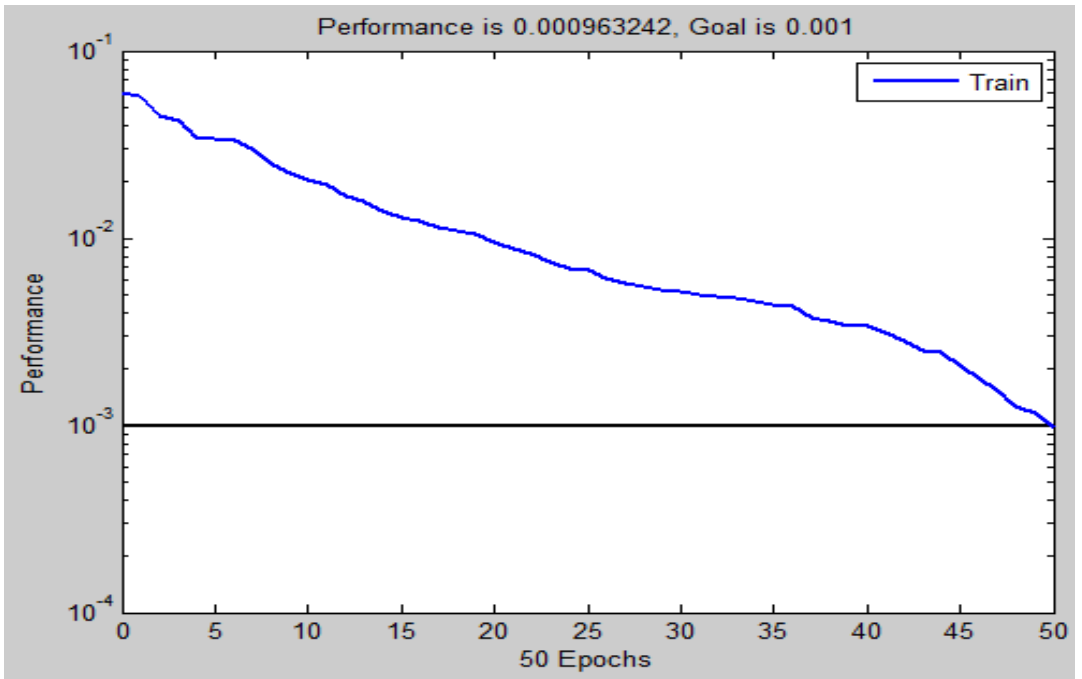

Figure 3. Error depression curve

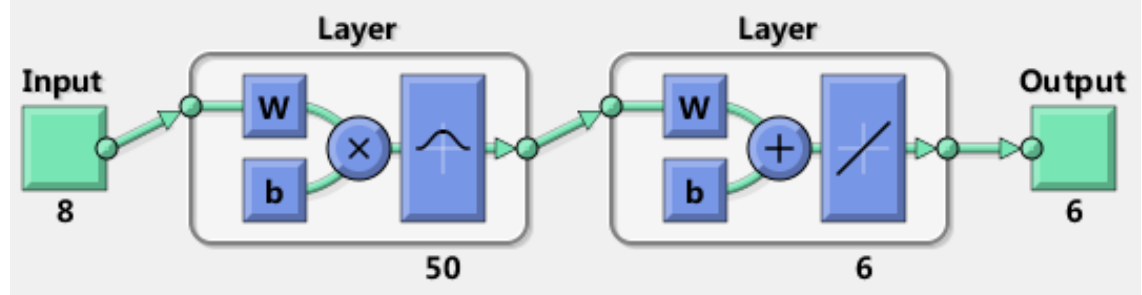

Figure 4. Structural Chart of Neural Network

Five sets of test samples were used to test the neural network, and the first-order test results were shown in Figure 5. It can be seen from the graph that the real values

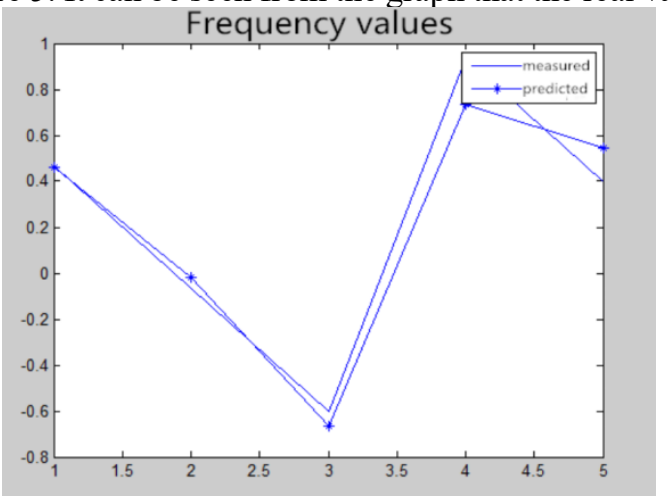

(a. Frequency values) of the five groups of samples are close to the predicted values, and the maximum error is 0.2 .

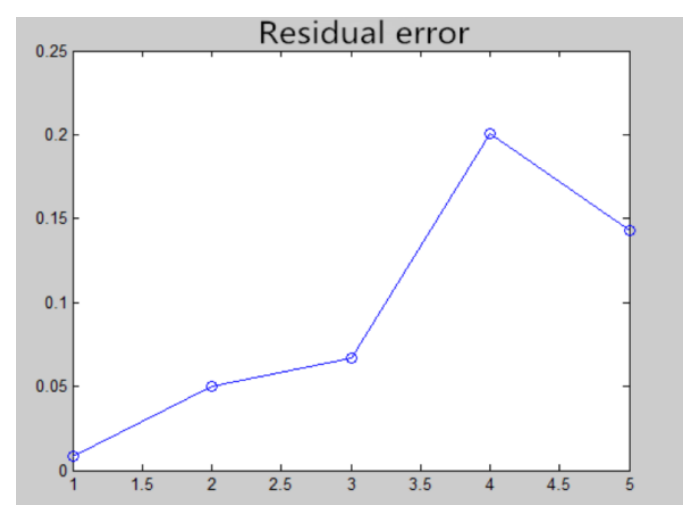

(b. Residual error)

Figure 5. Neural Network Testing Error

\subsection{Model Revision and Result}

The eighth-order frequencies of the bridge measured are input into the established radial basis function (RBF) neural network, and the output is the corrected values of the design parameters in the model updating. The corrected value is compared with the initial value, and the results are shown in Table 2. From the table, it can be seen that the change of design parameters before and after the modification is less than $5 \%$, which indicates that the modification results of the parameters have clear physical significance.

Table 2. Comparison of Initial and Corrected Design Parameters

\begin{tabular}{cccc}
\hline Design parameters & Initial value & $\begin{array}{c}\text { Correction } \\
\text { value }\end{array}$ & $\begin{array}{c}\text { Pre-and post- } \\
\text { correction deviation } \\
/ \%\end{array}$ \\
\hline$D_{1} / \mathrm{kg} \cdot \mathrm{m}^{3}$ & 2937 & 3011 & 2.5196 \\
$E_{1} / \mathrm{Pa}$ & $3.45 \times 10^{10}$ & $3.31 \times 10^{10}$ & -4.0580 \\
$D_{2} / \mathrm{kg} \cdot \mathrm{m}^{3}$ & 7850 & 8037 & 2.3822 \\
$E_{2} / \mathrm{Pa}$ & $2.05 \times 10^{11}$ & $1.97 \times 10^{11}$ & -3.9024 \\
\hline
\end{tabular}




\begin{tabular}{cccc}
\hline Design parameters & Initial value & $\begin{array}{c}\text { Correction } \\
\text { value }\end{array}$ & $\begin{array}{c}\text { Pre-and post- } \\
\text { correction deviation } \\
/ \%\end{array}$ \\
\hline$E_{3} / \mathrm{Pa}$ & $3.45 \times 10^{10}$ & $3.29 \times 10^{10}$ & -4.6377 \\
$E_{4} / \mathrm{Pa}$ & $3.25 \times 10^{10}$ & $3.15 \times 10^{10}$ & -3.0769 \\
\hline
\end{tabular}

The finite element model is modified according to the revised values of design parameters output by the neural network, and the model is revised. Modal analysis of the modified model is carried out to obtain the corresponding eighth-order analysis frequency. It is compared with the model analysis frequency and the measured frequency of arch bridge before revision, and the results are shown in
Table 4. The error between measured frequency $f_{E}$ and calculated frequency $f_{A}$ is calculated according to the following formula:

$$
E_{f}(\%)=\frac{f_{A}-f_{E}}{f_{E}}(\%)
$$

Table 3. Frequency comparison between measured frequencies and analysis frequencies before and after model modification

\begin{tabular}{|c|c|c|c|c|c|}
\hline frequency & $\begin{array}{c}\text { Measured value } \\
/ \mathrm{Hz}\end{array}$ & $\begin{array}{c}\text { Initial value } \\
/ \mathrm{Hz}\end{array}$ & $\begin{array}{l}\text { Initial error value } \\
\qquad / \%\end{array}$ & $\begin{array}{c}\text { Correction value } \\
/ \mathrm{Hz}\end{array}$ & $\begin{array}{c}\text { Error value } \\
\quad / \%\end{array}$ \\
\hline $\begin{array}{l}\text { First-order vertical } \\
\text { bending }\end{array}$ & 0.635 & 0.66916 & 5.3795 & 0.65431 & 2.8835 \\
\hline Two-step vertical bending & 0.874 & 0.87436 & 0.0412 & 0.87416 & 0.0183 \\
\hline $\begin{array}{l}\text { Three-step vertical } \\
\text { bending }\end{array}$ & 1.353 & 1.4036 & 3.7398 & 1.3874 & 2.5425 \\
\hline Five-step vertical bending & 2.847 & 2.521 & -11.4506 & 2.69174 & -6.2568 \\
\hline First-order torsion & 1.001 & 1.0306 & 2.9570 & 1.0255 & 2.4476 \\
\hline Two-order torsion & 1.984 & 2.0247 & 2.0514 & 2.02 & 1.8145 \\
\hline Three-order torsion & 2.91 & 3.0202 & 3.7869 & 2.9972 & 2.9966 \\
\hline Four-order torsion & 3.862 & 4.0178 & 4.0342 & 3.9227 & 1.5717 \\
\hline
\end{tabular}

It can be seen from the table that the relative errors between the analytical frequencies and the measured frequencies of each order after model modification are reduced, and the maximum error decreases from - $11.4506 \%$ to $-6.2568 \%$. The overall uniformity of the errors is better than that before model modification, and the accuracy of the model is further improved.

\section{Summary}

In this article, based on the measured frequency, the radial basis function (RBF) neural network method is used to modify the model. The main results are as follows:

(1) The eighth-order frequencies obtained from bridge measurement are selected as state parameters. Determining the network input of the nerve network. Based on sensitivity analysis method, six of the eight parameters of elastic modulus and density of each component of bridge structure are selected as design parameters to determine the output of the neural network.

(2) Using uniform design method, training samples and test samples with neural network are obtained by finite element calculation. Based on the neural network toolbox of MATLAB platform, the radial basis function (RBF) neural network is constructed. When the hidden layer center reaches 50 , the accuracy of the mean square error setting is achieved.

(3) When the trained neural network is input into the measured frequency parameters of the bridge, the output result is the optimal value of the design parameters. The output results are used to modify the initial finite element model. The modified model is obtained and modal analysis is carried out to obtain the corresponding modal frequency parameters. By comparing the frequencies of the model with the measured frequencies, the results show that the frequencies of the finite element model modified by the radial basis function neural network method are closer to the measured frequencies.

\section{Acknowledgments}

This research is supported by Shandong Key Laboratory of Appraisal and Retrofitting in Building Structures (Grant No. SZS201802), National Natural Science Foundation of China (Grant No. 51408261 and 51632003) and National Natural Science Foundation of Shandong Province (Grant No. ZR2017JL023).

\section{References}

1. Martin T. (2018) Neural Network Design. Machinery Industry Press, Beijing.

2. Zhang Zong qiang, Z.Q.(2014), Bearing capacity evaluation of Double-curvature Arch Bridge Based on static finite element model modified by RBF neural network. Lanzhou: Lanzhou Jiaotong University.,2014: 51-53

3. Chen Ming, M. (2013) Neural Network Principle and Example Elaboration of MATLAB. Tsinghua University Press, Beijing.

4. Kim G H, Park Y S. (2004) An improved updating parameter selection method and finite element model update using multiobjective optimisation 
technique[J]. Mechanical Systems and Signal Processing. , 18(1):59-78.

5. An jiu zhen, J.Z. (2013) Kang Bao long. Bridge Model Revision Based on Sensitivity Analysis [J]. Scientific and Technological Information., (11): 344345.

6. Xu Zhong an, Z.A. (2002) Wang Tian bao, Li Changying et al. Brief introduction of orthogonal experimental design method $[\mathrm{J}]$.Library and Information Guide., 12(5): 148-150.

7. Fang Kai tai, K.T. (1994) Uniform Design and Uniform Design Table. Science Publishing House, Beijing. 